\title{
Modelo de avaliação da gestão do conhecimento no processo de desenvolvimento do produto: aplicação em um estudo de caso
}

\author{
Sergio Luis da Silva \\ Prof. Dr. Depto. de Ciências da Informação e Programa de Pós-Graduação \\ em Engenharia de Produção da Universidade Federal de São Carlos - UFSCar \\ E-mail: sergiol@power.ufscar.br \\ Henrique Rozenfeld \\ Prof. Titular Núcleo de Manufatura Avançada da Escola \\ de Engenharia de São Carlos da Universidade de São Paulo - USP \\ E-mail:roz@sc.usp.br
}

\begin{abstract}
Resumo
O presente trabalho descreve a aplicação de um modelo de avaliação da Gestão do Conhecimento (GC) no processo de Desenvolvimento de Produtos (DP), focalizado no mapeamento da ocorrência de quatro tipos de conversões (do conhecimento] nos detalhamentos de quatro dimensões (deste processo). Este modelo foi construído com base no "estado da arte" da literatura Chipóteses e melhores práticas, que relacionam conversões do conhecimento com dimensões do DPJ. O modelo obtido mostrou-se significativo para a elaboração de uma visão ampla da situação em que se encontra a GC no processo de DP de uma empresa. A aplicação deste modelo, em um completo estudo de caso no DP de uma montadora do setor automotivo, mostrou sua viabilidade prática e especialmente sua utilidade em apontar lacunas na GC no processo de DP dessa empresa.
\end{abstract}

Palavras-chave

Gestão do conhecimento, desenvolvimento de produtos, diagnóstico organizacional.

\section{Evaluation model of knowledge management in the product development process: aplication in a case study}

\begin{abstract}
This paper describes an application of a model of Knowledge Management (KMJ evaluation in the Product Development (PD) process, mainly focused on mapping the occurrence of four types of (knowledge) conversion in some deployments of four dimensions lof this process]. This model was built supported by "state of the art" literature (hypothesis and best practices, that relates knowledge conversion with the PD dimensions]. The obtained model shows a significant effectiveness in elaboration of a wide view of KM situation embedded in PD process in a company. The application of this model in a complete case of $P D$ in an automotive assembler showed its practical viability and special usefulness in pointing out the KM gaps in the PD process of such a company.
\end{abstract}

Key words

Knowledge management, product development, organizational diagnosis. 


\section{INTRODUĈ̣̃O}

O presente trabalho está inserido tematicamente nas abordagens teóricas da Gestão do Conhecimento (GC) e do processo de Desenvolvimento de Produtos (DP) e, especificamente, concentra-se no estudo de determinados mecanismos para o trabalho com o conhecimento ao longo do processo de desenvolvimento de novos produtos.

O DP é considerado um dos mais importantes processos de negócio (business process) para a competitividade atual das empresas, sendo de fundamental importância para os crescentes esforços de adição de valor em sua capacidade de inovação (HARMSEN, 2000). Uma das condições básicas para o desempenho efetivo desse processo é o emprego disseminado da GC, que se manifesta de várias maneiras, como por exemplo, na capacidade de aprendizado e criatividade das pessoas envolvidas, na preocupação com a busca de conhecimentos e aprendizados externos à empresa, no armazenamento e divulgação dos conhecimentos adquiridos, dentre outras (LEONARD, 1995).

O modelo de avaliação proposto é constituído de dois temas, a GC e o processo de DP, que possuem um forte relacionamento orientador do foco do presente trabalho: o de se estudar o DP como um processo de negócio, constituído de quatro dimensões, onde podem ocorrer quatro tipos de conversões do conhecimento.

Baseando-se em abordagens teóricas consolidadas (PRASAD, 1996; CLAUSING, 1994; CLARK \& WHEELWRIGHT, 1992), pode-se afirmar que o processo de DP é constituído por quatro dimensões, que devem ser trabalhadas de forma integrada: a Estratégia (que envolve gestão do portfólio, avaliação do desempenho, relações interfuncionais e parcerias com fornecedores); a Organização (envolvendo a estrutura organizacional e a liderança, a cultura do trabalho em grupo e as condições de aprendizagem); as Atividades / Informações (o conjunto de atividades operacionais específicas executadas no processo de DP e as correspondentes informações manuseadas); e Recursos (as técnicas, métodos, ferramentas e sistemas utilizados para apoiar o DP).

Há uma contínua troca de conhecimentos nesses detalhamentos das quatro dimensões e a forma como isto ocorre influencia decisivamente a GC no processo de DP. Estas trocas ocorrem por meio de quatro conversões (NONAKA \& TAKEUCHI, 1997): conhecimentos transmitidos diretamente entre pessoas (Socialização); documentação dos conhecimentos (Externalização); agrupamento dos documentos (Combinação); e estudo dos documentos (Internalização).

Em um estudo bibliográfico orientado pelo "estado da arte" da literatura sobre GC e DP foram encontradas diversas referências que comprovam diretamente (melhores práticas) ou indiretamente (hipóteses) uma ou mais relações entre os detalhamentos das dimensões e as conversões do conhecimento. Apoiado neste estudo, o modelo de avaliação da GC no processo de DP foi construído, estruturado na análise e investigação dos relacionamentos entre conversões e dimensões ao longo deste processo.

Portanto, o objetivo principal deste artigo é apresentar o modelo de avaliação e mostrar sua viabilidade prática, basicamente buscando respostas para a seguinte pergunta: Como este modelo pode ser aplicado na avaliação prática da GC em um processo de DP de uma empresa?

A principal justificativa para a realização deste trabalho reside no fato de que o relacionamento entre DP e GC, embora percebido e comentado na literatura, vem sendo tratado de forma fragmentada e pouco sistemática, não existindo ainda um modelo que permita às pessoas e empresas avaliar e a partir disto gerenciar melhor esta relação.

Ao se propor um modelo que avalia a situação, a partir da geração de resultados na forma de um "mapa" detalhado, que pode subsidiar decisões futuras na GC no DP da empresa, este trabalho justifica sua aplicação e utilidade prática. Muitas empresas já reconhecem a importância do tema GC, e em particular no DP, e também possuem acesso a inúmeras propostas (organizacionais, tecnológicas, etc.) que prometem solucionar os problemas relacionados ao tema. No entanto, tais empresas normalmente têm dificuldades para delimitar e caracterizar esses problemas e, a partir disso, tomar suas decisões. Dessa forma, o modelo proposto pretende contribuir para a superação dessas dificuldades.

O modelo de avaliação da GC no DP é esquematizado e explicado no próximo tópico, em que é mostrado seu potencial de aplicação. Os dois eixos principais que formam o modelo, o DP e suas dimensões e a $\mathrm{GC}$ e as conversões do conhecimento, também são sucintamente explicados neste tópico.

A metodologia de pesquisa que estrutura este estudo é colocada em seus pontos principais no tópico Metodologia. A aplicação do modelo, com o estudo de caso realizado (descrição, resultados e discussão), é apresentada no tópico Estudos de caso realizados. Finalmente, o artigo encerra-se com as conclusões sobre o trabalho.

\section{MODELO DE AVALIAC̣̃̃O DA GESTÃO DO CONHECIMENTO NO PROCESSO DE DESENVOLVIMENTO DE PRODUTOS}

A construção do referido modelo é feita no subtópico Construção do modelo, explicando-se como os relacionamentos entre dimensões e conversões foram trabalhados 
de forma a se construir o modelo de avaliação. As bases teóricas sobre DP (e suas dimensões) e GC (conversões do conhecimento), que formam o contexto teórico necessário à concepção do modelo, são expostas nos outros dois subtópicos.

\section{Construção do Modelo}

Conforme anteriormente exposto na Introdução, hipóteses e melhores práticas acenam para a existência de relacionamentos entre as conversões do conhecimento e as dimensões do DP, o que sustenta a principal idéia ou proposição deste trabalho, de que a análise das conversões, em cada uma das dimensões, pode ser usada para investigar como os conhecimentos são manuseados ou trabalhados em todo o processo de DP. Esta proposta pode, então, resultar em um efetivo modelo de avaliação da GC.

Analisando-se em detalhes vários dos trabalhos (autores) anteriormente e posteriormente citados, pode-se levantar hipóteses sobre a existência de relacionamentos entre as dimensões do DP e as conversões do conhecimento. Por exemplo, a hipótese de que alguns procedimentos podem otimizar a externalização nas atividades do DP, advindo da interpretação das propostas de Kleiner \& Roth (1997) e Sobek II et al. (1998) sobre design learning history (registros de aprendizados - lessons learned - ocorridos durante o projeto). Diversas hipóteses construídas com a mesma lógica do exemplo citado podem ser feitas para vários dos relacionamentos entre dimensões e conversões.

por Bolisani \& Scarso (1999) e Baba \& Nobeoka (1998), no sentido de usar o recurso CAD 3-D do DP para otimizar a ocorrência das conversões do conhecimento.

O conjunto das várias hipóteses e melhores práticas permite que se investigue como os conhecimentos são manuseados ou trabalhados ao longo de todo o processo de DP e, portanto, constitui-se em um mecanismo efetivo de avaliação da GC no DP. Esse relacionamento percebido entre as duas grandes linhas ou eixos teóricos do presente trabalho acena para a pertinência de que uma avaliação ampla da GC no DP possa fazer uso de um modelo que relacione as dimensões deste processo com as conversões do conhecimento.

Os mencionados relacionamentos entre dimensões e conversões, pontualmente percebidos por meio das hipóteses e melhores práticas, também são reforçados por reflexões amplas em cada um desses eixos teóricos, as quais mostram o potencial de relacionamento entre eles. Uma reflexão relevante neste sentido é que o DP, ao ser tratado como um processo, alarga as atribuições das áreas funcionais e envolve pessoas de diferentes áreas (valorizando o conhecimento heterogêneo), amplia a participação de clientes e fornecedores no projeto (valorizando o conhecimento externo), e melhor integra e coordena as atividades do projeto, o que, segundo Carrillo \& Gaimon (2000), relaciona esse processo com a criação e acumulação do conhecimento na empresa, e com sua circulação e compartilhamento (BROWN \& DUGUID, 2000; EL SAWY, 2001).

$\mathrm{O}$ modelo de avaliação da GC

\section{A principal idéia ou proposição deste trabalho: a análise das conversões, em cada uma das dimensões, pode ser usada para investigar como os conhecimentos são manuseados ou trabalhados em todo o processo de DP.} no DP parte do pressuposto (verdadeiro na maioria dos casos) de que já existem iniciativas de conversões do conhecimento no processo de DP. O foco está em considerar estas iniciativas e mapeálas, entendendo como são feitas e detectando lacunas existentes (tanto conversões que estão sendo mal realizadas como as que não estão ocorrendo em certos

Somando-se às hipóteses, elaboradas pelo autor do presente artigo, com base em sua interpretação do conteúdo das referências sobre DP, há também diversos autores e trabalhos específicos que detectaram e descreveram diretamente a existência de relacionamentos entre certas dimensões do DP e a GC e, em muitos casos, especificamente com as conversões do conhecimento, usando uma terminologia igual ou equivalente à adotada na presente pesquisa. Esses trabalhos são aqui denominados de melhores práticas. Apenas para exemplificar, podem ser mencionadas as melhores práticas apontadas detalhamentos das dimensões do DP, tentando-se encontrar explicações para isso).

A Tabela 1 sintetiza o referido modelo, em termos de seu conjunto constituinte de hipóteses e melhores práticas, que serão verificadas quanto as sua ocorrências no estudo de caso apresentado no tópico Estudos de Caso realizados, que permitiu o completo teste deste modelo.

\section{Desenvolvimento de Produtos e suas Dimensões}

As abordagens que tratam do DP provêm de diferentes áreas, inter-relacionadas, porém, com focos específicos. 
Tabela 1: Modelo de avaliação da GC no processo de DP.

\begin{tabular}{|c|c|c|c|c|}
\hline \multirow{2}{*}{$\begin{array}{l}\text { DIMENSÕES DO DP (NÚMERO DE } \\
\text { DETALHAMENTOS NA DIMENSÃO) } \\
\text { (VER SUBTÓPICO) }\end{array}$} & \multicolumn{4}{|c|}{ CONVERSÕES DO CONHECIMENTO (VER SUBTÓPICO) } \\
\hline & SOCIALIZAÇÃO & EXTERNALIZAÇÃO & COMBINAÇÃO & INTERNALIZAÇÃOO \\
\hline Estratégia (4) & $10 \mathrm{HT} 1 \mathrm{MP}$ & ЭНТ २МР & 9НТ 1MP & $10 \mathrm{HT} 1 \mathrm{MP}$ \\
\hline Organização (4) & 12HT 6MP & $11 \mathrm{HT}$ 6MP & 8НТ 2MP & 12HT 4MP \\
\hline Atividades e Informação (13) & 26HT 2MP & 34HТ 2MP & 21HT & 21HT 1MP \\
\hline Recursos (7) & 9НТ ЗМР & 19HТ ЗМР & 9HТ 2MP & 13НТ ЗМР \\
\hline
\end{tabular}

Obs.: HT = hipóteses; MP = melhores práticas. Por exemplo: a informação 10HT 1MP, na célula referente ao cruzamento entre Socialização versus Estratégia, significa que no modelo foram formuladas dez hipóteses e uma melhor prática que atestam a ocorrência dessa conversão do conhecimento nos detalhamentos dessa dimensão (nas quatro perspectivas da Estratégia, expostas no sub-tópico seguinte). 0 mesmo raciocínio vale para as demais células.

No presente trabalho, a abordagem predominante é proveniente da engenharia e da administração, tendo como principais autores: Prasad (1996; 1997), e Pugh (1996), mais ligados à área de engenharia, e Clark \& Fujimoto (1991), Clark \& Wheelwright (1992), e Clausing (1994), mais relacionados à administração e à gestão. Os referidos autores normalmente relacionam suas proposições teóricas com o que é observado nas melhores práticas das empresas de classe mundial.

O DP é um processo tipicamente interfuncional, em que as interfaces entre funções se fazem cada vez mais de forma paralela e com rapidez na troca de informações (DAVENPORT, 1994). O conceito de processo de negócio substitui a clássica visão funcional por uma visão horizontal, em que a unidade de análise passa a ser uma cadeia de atividades/eventos.
Segundo Davenport (1994, p.7), "um processo é uma ordenação específica das atividades de trabalho no tempo e no espaço, com um começo, um fim, e inputs e outputs claramente identificados". De forma similar, Rozenfeld et al. (2000, p.16) afirmam que "o processo de negócio representa o conjunto de atividades, associadas às informações que manipula, utilizando os recursos e a organização da empresa. Forma uma unidade coesa e está direcionado a um determinado mercado/cliente, com fornecedores bem definidos". Um processo de negócios é algo que ocorre naturalmente nas empresas, não sendo percebido, em muitos casos, por estar ofuscado pela estrutura funcional predominante.

Seguindo esta abordagem de processo, interessa para o foco do presente trabalho detalhar esse conjunto agrupado em quatro dimensões, apresentando-se os

Figura 1: Elementos ou dimensões do processo orientados por uma visão ou imagem única (adaptado de ROZENFELD et al., 2000]

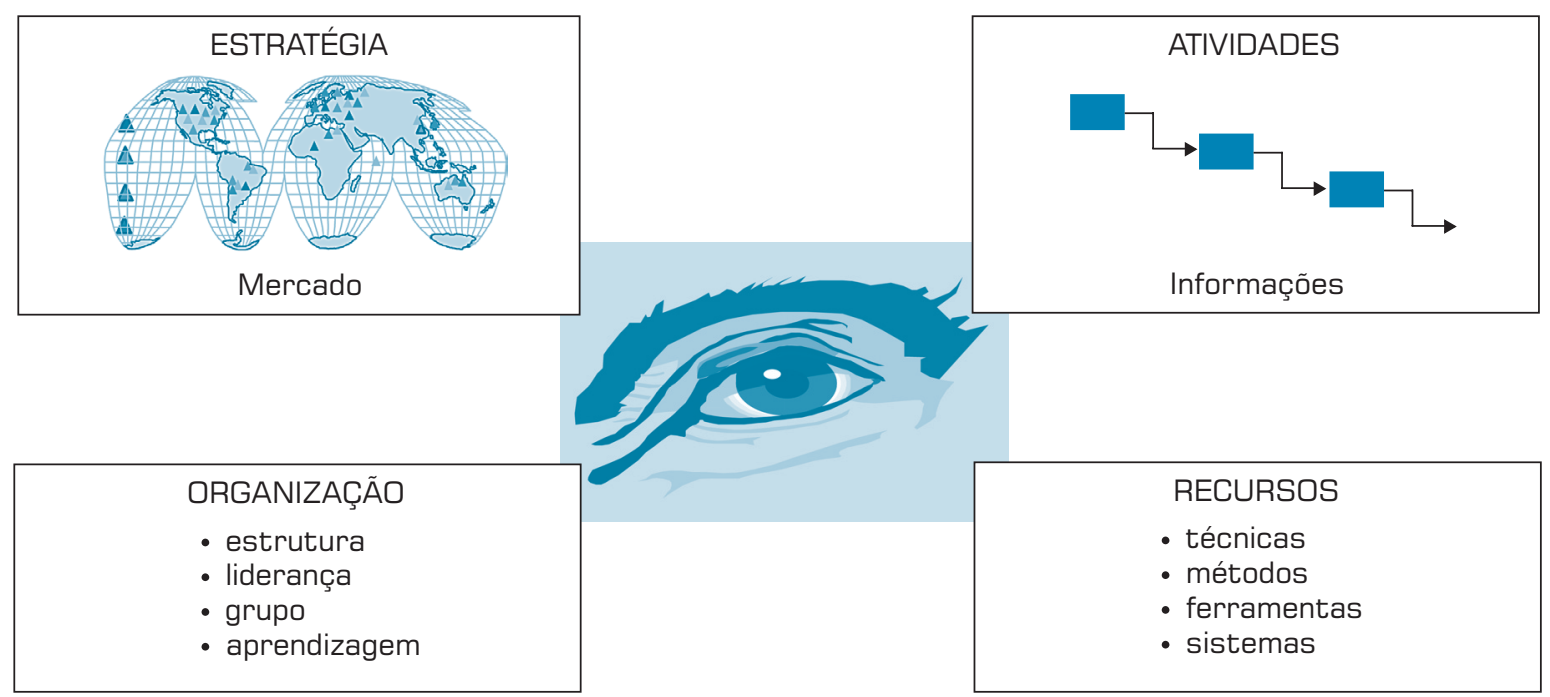


mais citados (e empregados) pela literatura de DP. A grande contribuição está em se discutir problemas específicos inseridos nestes elementos ou dimensões sem perder a visão do todo (Figura 1), a "imagem única", dada pelo processo (ROZENFELD et al., 2000).

A dimensão Estratégia do DP, baseando-se principalmente em CLARK \& FUJIMOTO (1991), CUSUMANO \& NOBEOKA (1998) e ROBERTSON \& ULRICH (1998), pode ser analisada sob as perspectivas de:

1. Gestão ou administração do portfolio de produtos (projetos): coordenação conjunta dos projetos em andamento ou em planejamento, também coordenando estrategicamente as relações inter-projetos;

2. Avaliação do desempenho do processo de DP: avaliação de projetos de DP (produtos desenvolvidos) em relação ao portfolio de projetos da empresa;

3. Condução das alianças e parcerias para o DP: realização de integração inter-organizacional envolvendo fornecedores, clientes, instituições, etc.;

4. Condução das relações interfuncionais/interdepartamentais: realização de integração em nível estratégico entre marketing, engenharia e manufatura.

A dimensão Organização do DP, baseando-se principalmente em CLARK \& FUJIMOTO (1991) e ROZENFELD et al. (2000), pode ser analisada sob as perspectivas de: 1. Adoção e manutenção da estrutura organizacional para o $D P$, envolvendo as escolhas entre estrutura funcional, gerenciamento "peso leve" ou "peso pesado", e time autônomo;

2. Execução do trabalho de liderança no $D P$, que se refere às habilidades e ao comportamento do gerente de produto;
A dimensão Atividades/Informações do DP trata das diversas atividades que ocorrem ao longo do processo de DP, e das informações que são por ela trocadas, com maior ou menor paralelismo ou simultaneidade. Diversos autores descrevem e detalham estas atividades. Clark \& Wheelwright (1992) são um dos que de forma mais completa fazem isso. Existem ainda propostas institucionais como o modelo da APQP (Advanced Product Quality Planning) derivado da QS 9000, voltado ao setor automotivo. Sintetizando todas estas abordagens, têmse as seguintes atividades (treze) para esta dimensão:

1. Pesquisa de mercado, levantamento das possibilidades tecnológicas e determinação dos requisitos dos clientes;

2. Identificação de riscos, avaliação de viabilidade e planejamento de recursos;

3. Tradução do conceito do produto em estilo, layout, componentes e especificações;

4. Envolvimento de fornecedores no processo de DP;

5. Construção de modelos físicos e avaliação de estilo e layout;

6. Transformação de resultados das etapas anteriores em desenho e normas;

7. Elaboração, construção e testes de protótipos;

8. Tradução das especificações do projeto do produto no desenvolvimento do projeto do processo (fabricação e montagem);

9. Realização de produção piloto e testes de validação do produto;

10.Emprego de gates para avaliação do andamento do projeto; 11.Normalização do conteúdo das informações;

12.Normalização do formato das informações;

13. Controle de atualizações e armazenamento das informações.

\section{- m termos conceituais, o DP é um} — processo essencialmente criador e disseminador de novos conhecimentos, e por este motivo a teoria da criação do conhecimento de Nonaka \& Takeuchi é a mais adequada para investigar a GC nesse processo
Os recursos utilizados no DP compõem-se de métodos, técnicas, ferramentas e sistemas que podem ser aplicados como apoio em uma ou mais das dimensões anteriores, especialmente nas Atividades/Informações. De Clark \& Wheelwright (1992), sintetizando vários autores, e também de ROZENFELD et al.(2000), podem ser listados os seguintes recursos:

1. O método de QFD (Quality

3. Execução do trabalho em grupo, em uma cultura que estimule a comunicação e a gestão de conflitos;

4. Existência de programas de capacitação e de acompanhamento da qualificação do pessoal envolvido com o $D P$, que incrementam a aprendizagem organizacional neste processo.
Function Deployment), para o levantamento das necessidades e desejos do cliente e sua tradução em especificações de engenharia;

2. As técnicas de DFMA (Design for Manufacturing and Assembly), para desenvolvimento de um projeto voltado à capacidade da manufatura da empresa (fabricação e montagem); 
3. As ferramentas e sistemas CAD-CAE-CAM e CAPP, respectivamente Computer Aided Design-EngineeringManufacturing e Computer Aided Process Planning, usadas de forma integrada ou não, para representar o produto em desenhos, cálculos de engenharia e instruções de fabricação (sendo seu uso dependente do setor industrial);

4. Os recursos de FMEA (Failure

Mode Effect Analysis) de produto e de processo (fabricação), Análise/Engenharia do Valor, e técnicas de fabricação e uso de protótipos (funcionais e não-funcionais), para, respectivamente, a análise de falhas no projeto e na fabricação, a melhora de soluções disponibilizadas pelo produto, e a orientação para o teste e visualização de produtos em desenvolvimento;

5. Os sistemas PDM/EDM, respectivamente Product/Engineering Data Management, integrados ou não aos PM (Project Management) dos sistemas ERP (Enterprise Resource Planning), para o gerenciamento e controle das informações utilizadas e geradas pelas etapas do projeto de DP;

6. Os recursos de TI para apoio ao trabalho em grupo, busca de conhecimentos externos e para o treinamento e ensino, recursos estes que servem para facilitar o trabalho individual e coletivo dos projetistas;

7. As ferramentas para a construção (desenho) e manutenção de modelos de referência, que facilitam a representação e o emprego da visão de processos no DP.

Se neste item o foco principal foi o DP como processo e suas quatro dimensões, o próximo trata da GC focalizando a abordagem das conversões do conhecimento.

\section{Gestão do Conhecimento e suas Conversões}

Nos anos 80, o tema conhecimento tornou-se mais presente devido ao advento das abordagens teóricas relacionadas à sociedade do conhecimento, ao aprendizado organizacional e às competências essenciais na gestão estratégica (SPENDER, 1996). Em anos recentes, as pesquisas nestas abordagens intensificaram suas aplicações práticas e o aprofundamento teórico, focalizando a necessidade de se entender como as organizações trabalham com o conhecimento para desenvolver novos produtos, novos processos e novas formas ou arranjos organizacionais mais flexíveis, proporcionando uma vantagem competitiva sustentável (HAYES \& PISANO, 1994).

Também tornou-se relevante entender como as organizações trabalham com o conhecimento especializado, sem perder o foco em uma visão unificada do todo, caracterís- tica de uma visão de processos de negócios (DRUCKER, 1988; QUINN, 1999). Mesmo pensando em todos os processos da empresa, esses autores mencionam que começar a GC pelo processo de DP, devido a sua inerente dependência quanto ao conhecimento, é uma das melhores formas de se iniciar um trabalho com este tema.

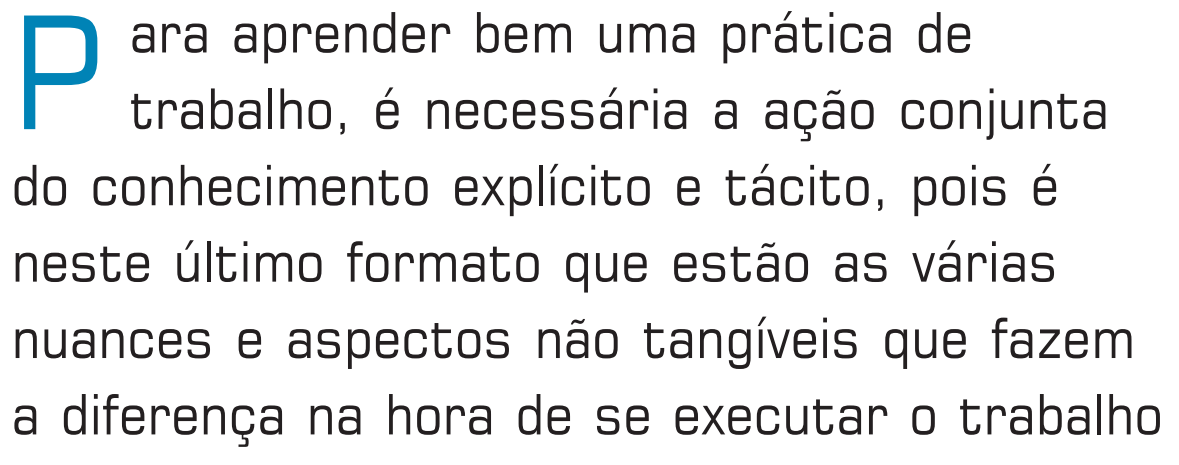

Das várias abordagens teóricas relacionadas à GC, a que mais proximidade ou adequação possui com a necessidade de se entender a GC no DP é a teoria apresentada na escola da criação do conhecimento (Knowledge Creation) de Nonaka \& Takeuchi (1997). Isso porque, em termos conceituais, o DP é um processo essencialmente criador e disseminador de novos conhecimentos, o que é o foco principal dessa teoria e, em termos empíricos, porque Nonaka \& Takeuchi (1997) construíram e testaram sua teoria tendo como base inovações (novos produtos) executadas em casos de DP considerados exemplares e bem-sucedidos. Portanto, como a proposta do presente trabalho trata detalhadamente o DP, a teoria da criação do conhecimento é a mais adequada para investigar a GC nesse processo, viabilizando a construção do modelo de avaliação.

Particularmente importante para se entender a GC, e em especial a teoria da criação do conhecimento, é entender o conhecimento como sendo constituído por dois formatos. Há um amplo consenso de que o conhecimento existe no formato tácito (habilidades e experiência inerentes a uma pessoa, difícil de ser formalizado e transferido a outra pessoa) e no formato explícito (conhecimento relativamente fácil de se codificar em textos, gráficos, tabelas, figuras, desenhos, esquemas, etc. e transferir), sendo que os autores mais relevantes na definição e uso destes formatos (NONAKA \& TAKEUCHI, 1997) consideram que um trabalho efetivo com o conhecimento somente é possível em um ambiente onde possa ocorrer a contínua conversão entre estes dois formatos através de quatro modos:

- Socialização - uma frase síntese da conversão do conhecimento tácito de uma pessoa no conhecimento tácito de 
outra pessoa é a "troca de conhecimentos face a face entre as pessoas" (Figura 2). Para isso ocorrer, são necessários diálogos freqüentes, brainstorming, trabalho do tipo "mestre-aprendiz", observação e imitação, compartilhamento de experiências, etc.

- Externalização - uma frase síntese da conversão do co- nhecimento tácito do indivíduo em algum tipo de conhecimento explícito é o "registro do conhecimento da pessoa feito por ela mesma" (Figura 3). Para isso ocorrer, são necessários relatos orais ou visuais, via metáforas, analogias, modelos, textos, imagens, figuras, regras, etc.

- Combinação - uma frase síntese da conversão de algum

Figura 2: Exemplos de hipóteses e melhores práticas, aleatoriamente escolhidas de duas dimensões do processo de DP, que indicam a ocorrência da Socialização.

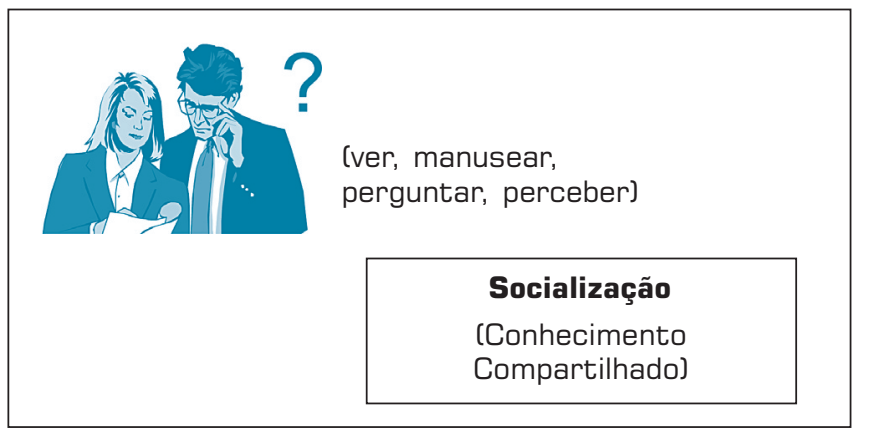

\begin{tabular}{|c|c|}
\hline Estratégia & $\begin{array}{ll}\cdot & \text { Socialização } \\
\cdot & \\
\cdot & \end{array}$ \\
\hline $\begin{array}{l}\text { Avaliação do } \\
\text { desempenho } \\
\text { do processo } \\
\text { de DP }\end{array}$ & $\begin{array}{ll}\cdot & \text { (HT) reuniões } \\
. & \text { para auditoria } \\
\cdot & \text { e/ou } \\
. & \text { workshops } \\
& \text { pós-projeto }\end{array}$ \\
\hline
\end{tabular}

\begin{tabular}{|c|c|}
\hline Atividades & Socialização \\
\hline $\begin{array}{l}\text { Elaboração, } \\
\text { construção e } \\
\text { testes de } \\
\text { protótipos }\end{array}$ & $\begin{array}{l}\text { (MP) discussões sobre } \\
\text { protótipos são } \\
\text { potenciais catalisadores } \\
\text { de trocas de } \\
\text { conhecimento tácito }\end{array}$ \\
\hline
\end{tabular}

Figura 3: Exemplos de hipóteses e melhores práticas, aleatoriamente escolhidas de duas dimensões do processo de DP, que indicam a ocorrência da Externalização.

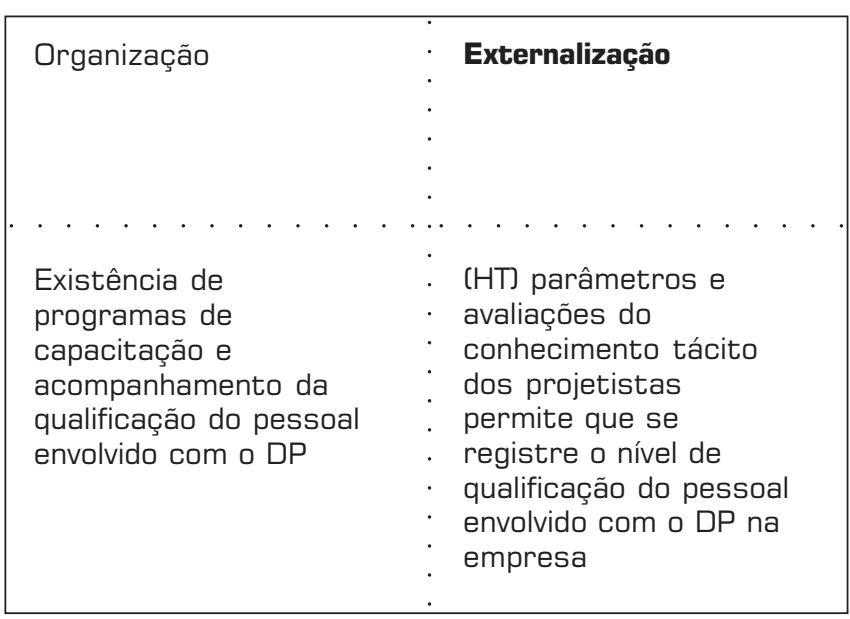


tipo de conhecimento explícito gerado por um indivíduo para agregá-lo ao conhecimento explícito da organização é o "agrupamento dos registros do conhecimento" (Figura 4), que ocorre por meio do agrupamento e processamento de diferentes conhecimentos explícitos.

- Internalização - uma frase síntese da conversão de alguma parte do conhecimento explícito da organização em conhecimento tácito do indivíduo é o "aprendizado pes-

Figura 4: Exemplos de hipóteses, aleatoriamente escolhidas de duas dimensões do processo de DP, que indicam a ocorrência da Combinação.

\begin{tabular}{|c|c|}
\hline Estratégia & Combinação \\
\hline $\begin{array}{l}\text { Gestão ou Administração do } \\
\text { portfólio de produtos (projetos) }\end{array}$ & $\begin{array}{l}\text { [HT] formas unificadas de } \\
\text { agrupamento de documentos } \\
\text { dos projetos }\end{array}$ \\
\hline
\end{tabular}

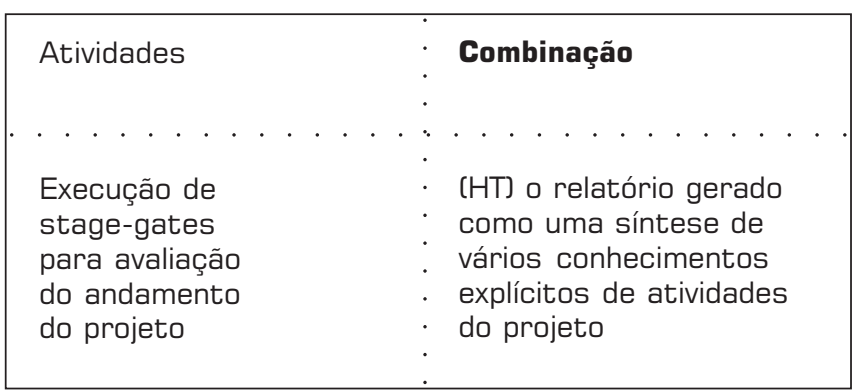

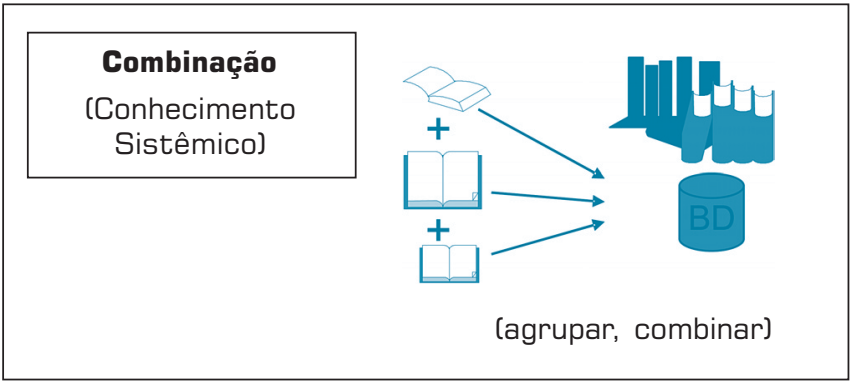

Figura 5: Exemplos de hipóteses, aleatoriamente escolhidas de duas dimensões do processo de DP, que indicam a ocorrência da Internalização.

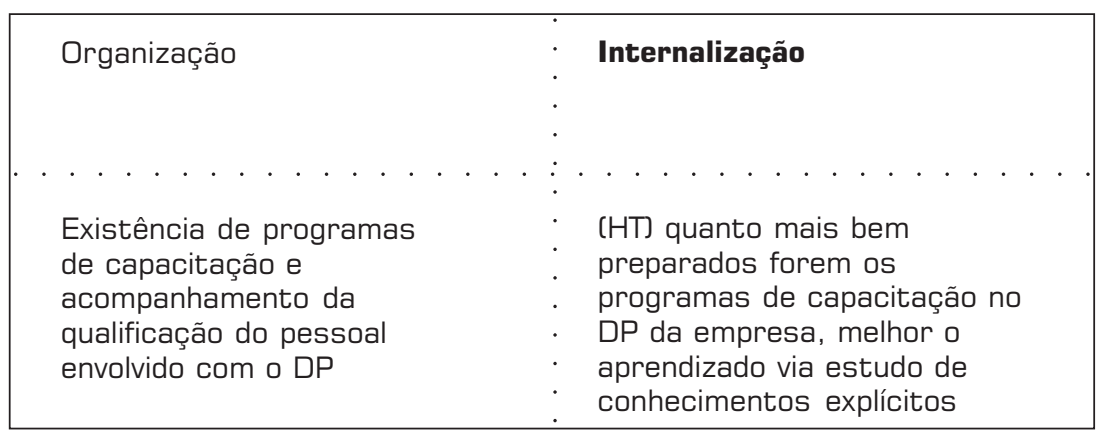

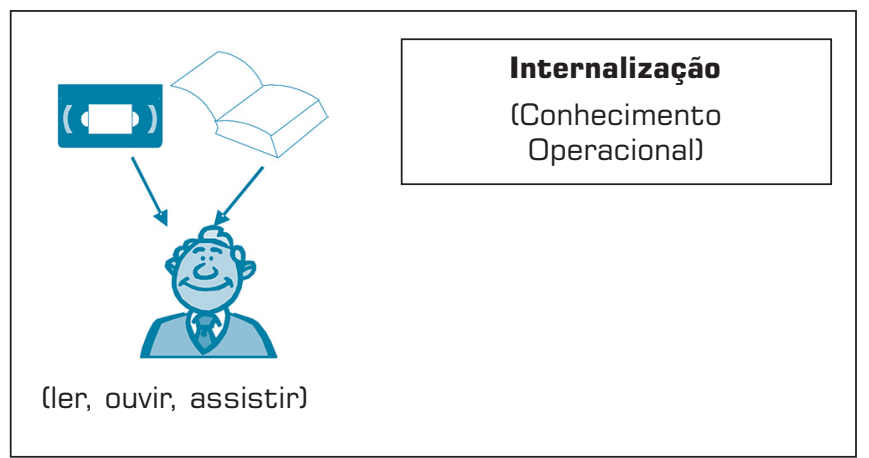

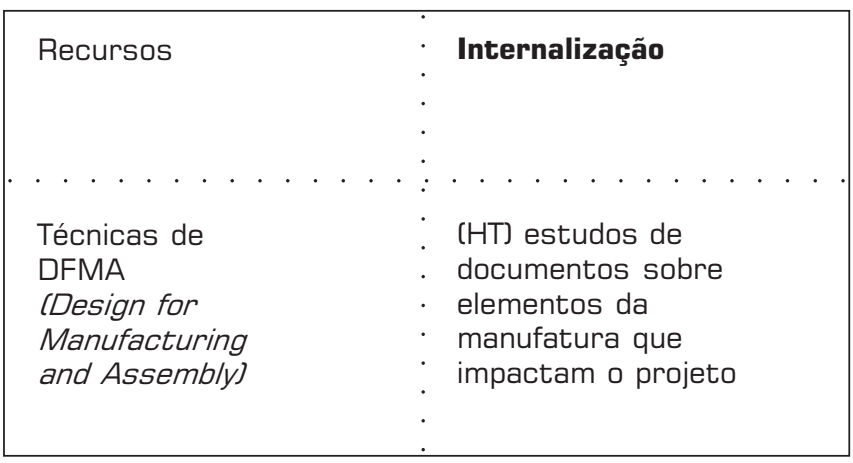


soal a partir da consulta aos registros do conhecimento" (Figura 5). Para isso ocorrer, é necessário a leitura/visualização e o estudo, individual, de conhecimentos explícitos registrados em diferentes tipos de documentos (textos, imagens, etc.)

Os ciclos de conversão do conhecimento, passando várias vezes por estes quatro modos, formam uma espiral que serve para analisar e entender os mais diversos casos de criação e disseminação do conhecimento (NONAKA \& TAKEUCHI, 1997), sendo que cada caso terá suas particularidades ou especificidades (Figura 6). Basicamente, este tipo de análise com as quatro conversões é a que é feita para cada perspectiva da Estratégia e da Organização, cada Atividade e Recurso, do processo de DP.

Para a transferência do conhecimento tácito são necessárias fortes ligações entre pessoas em espaços coletivos, tornando-se prioritária a relação "face a face". Já a transferência do conhecimento explícito pode se dar sem esta relação, podendo ser completada totalmente pelo uso, por exemplo, de meios da TI como uma intranet (CLIFFE, 1998).

Sutton \& Pfeffer (1999) afirmam que, para aprender bem uma prática de trabalho, é necessária a ação conjunta do conhecimento explícito e tácito, pois é neste último formato que estão as várias nuances e aspectos não tangí- veis que fazem a diferença na hora de se executar o trabalho. O exemplo que estes autores dão é bastante ilustrativo desta questão: Há inúmeros livros, figuras, filmes, relatos de visitas a fábricas, enfim, conhecimento no formato explícito, sobre o sistema Toyota de produção (similar à produção enxuta). Entretanto, apenas com um trabalho conjunto ou estágio duradouro junto aos experientes engenheiros e técnicos nas fábricas da Toyota, ou a permanência destes por um certo período de tempo na empresa que pretende implantar este sistema de produção, enfim, criando-se oportunidades de contato com o conhecimento no formato tácito, é que é possível captar e incorporar efetivamente os conhecimentos sobre este sistema.

A discussão sobre o DP e suas dimensões e sobre a GC e as conversões do conhecimento formam os dois eixos principais do modelo já exposto. O tópico seguinte apresenta brevemente a metodologia utilizada na construção e na aplicação deste modelo, cujos resultados serão apresentados no tópico Estudos de Caso realizados.

\section{METODOLOGIA}

A pesquisa feita neste trabalho emprega o método hipotético-dedutivo (DANE, 1990), à medida que parte

Figura 6: Espiral dos modos de conversão do conhecimento (tácito-explícito) (adaptado de NONAKA \& TAKEUCHI, 1997].

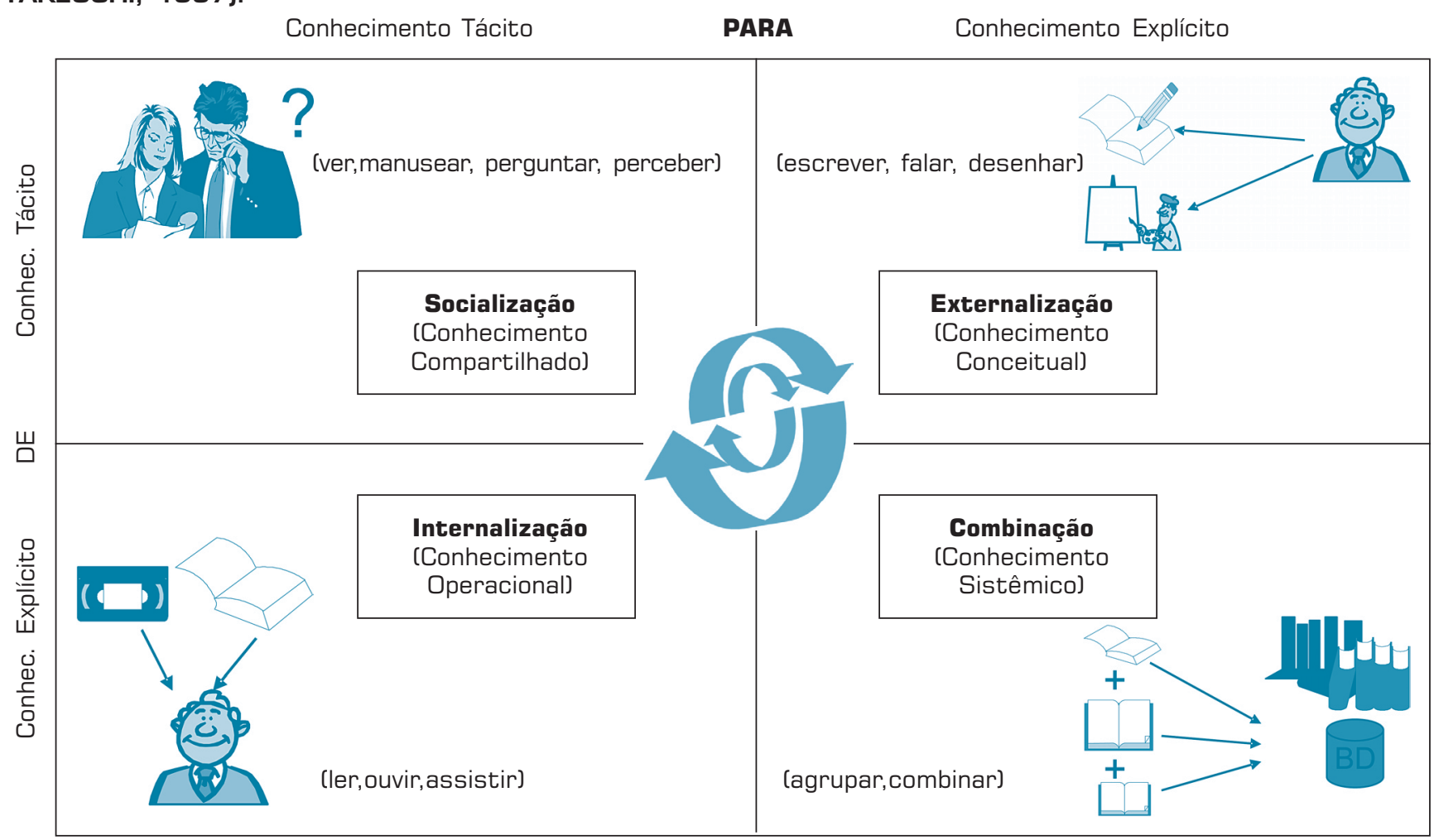


de teorias sobre o processo de DP e sobre a GC, descartando aquelas que não servem a presente pesquisa, e buscando a contínua refutação do modelo construído ao submetê-lo a testes rigorosos por meio de sua aplicação prática em empresas.

O problema de pesquisa é abordado principalmente de forma qualitativa e descritiva, pois visa descrever e interpretar as características de determinado fenômeno (os relacionamentos existentes entre os detalhamentos das dimensões do DP com as conversões do conhecimento), definindo-os e delimitandoos com a ajuda de um modelo de avaliação proposto.

Em termos de método de procedimento de pesquisa, o presente trabalho emprega o estudo de caso pois este é adequado à necessidade de se testar o modelo de avaliação da GC no DP, verificando as teorias empregadas na construção deste modelo (LAKATOS \& MARCONI, 1995; YIN, 1994). Além disso, o estudo de caso é o mais recomendado para descrever e avaliar situações quando a questão de pesquisa é do tipo "como" "Como este modelo pode ser aplicado na avaliação prática da GC em um processo de DP de uma empresa?" e quando o pesquisador não tem nenhum controle sobre o evento.

Ao se decidir pelo uso do estudo de caso, deve-se ter clara a principal limitação deste método: a impossibilidade de que seus resultados sejam generalizados para uma população ou universo (generalização estatística). Esta limitação não compromete a contribuição deste trabalho - que é a de propor um modelo para avaliação da GC no DP -, pois o estudo de caso permite que os resultados do teste deste modelo sejam generalizados em termos de proposição teórica (generalização analítica). Levando-se em consideração esta característica, foram escolhidos casos para estudo de forma não probabilística e intencional, por meio da seleção daqueles que mais riqueza de detalhes e complexidade podem oferecer ao teste do modelo proposto, permitindo, assim, atingir a generalização teórica ou analítica desejada.

Para operacionalizar as escolhas metodológica feitas para este trabalho, foi empregado como instrumento de pesquisa principal um questionário com perguntas abertas e fechadas aplicado por meio de entrevistas com pessoas envolvidas com o processo de DP, de diferentes especialidades, tempo de experiência com o DP e níveis hierárquicos.

O questionário é basicamente o conjunto das hipóte- ses e melhores práticas que formam o modelo (sintetizado na Tabela 1 anteriormente apresentada), e que são verificadas quanto a sua ocorrência pelos entrevistados, também com alguma apreciação ou julgamento quanto à forma ou maneira como ocorrem. Assim, pode-se chegar a conclusões quanto à própria ocorrência das con-

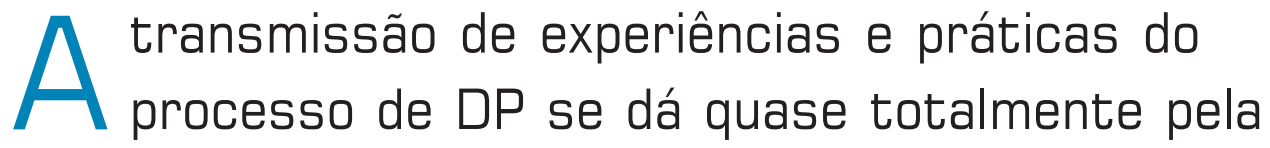
via tácita e, portanto, é fortemente dependente da Socialização, com vantagens (facilidade e rapidez) e desvantagens (monopólio individual do perda da memória corporativa]

versões do conhecimento, e portanto da GC, nas dimensões do processo de DP. O conjunto completo de hipóteses e melhores práticas, devido a sua extensão, não cabe nos limites de espaço do presente artigo, mas pode ser consultado integralmente em Silva (2002).

$\mathrm{Na}$ aplicação desse questionário, primeiro há um diálogo com o entrevistado quanto a sua relação com o DP da empresa, o que permite ao entrevistador adquirir uma percepção inicial do envolvimento direto e indireto do entrevistado com o processo de DP e com suas dimensões específicas, e onde se explica o propósito da pesquisa e o significado das dimensões do DP e das conversões do conhecimento. Por fim, é feita a coleta das informações desejadas, com o pesquisador conduzindo o preenchimento, esclarecendo dúvidas, e questionando as nuances das respostas do entrevistado quanto às formas de ocorrência das hipóteses e melhores práticas no processo de DP da empresa.

\section{ESTUDOS DE CASO REALIZADOS: TESTANDO O MODELO}

Uma versão preliminar do modelo foi testada pioneiramente (piloto) em um ambiente físico simulado de DP dado por uma Fábrica Integrada Modelo (FIM). Esse espaço, um laboratório de pesquisa e ensino de engenharia, dentro da universidade, tem todos os elementos necessários para ser um ambiente bem próximo do real, tais como: produto (do setor metal-mecânico; um redutor universal), pessoas (personagens criados com uma posição profissional e responsabilidades), recursos (máquinas, computadores, etc.), entre outros. A aplicação do 
modelo de avaliação da GC no cenário de DP da FIM, onde professores e alunos foram entrevistados, mostrou sua viabilidade prática e efetividade em mapear a situação do processo de DP, em termos de criação, armazenamento e disseminação do conhecimento. Como limitações dessa primeira aplicação deve-se mencionar que foi feita em um ambiente simulado de DP, e portanto com seus parâmetros controlados e onde não estão presentes todas as instabilidades típicas de um ambiente real. Mais detalhes dessa aplicação podem ser encontrados em Silva \& Rozenfeld (2001a).

A primeira aplicação do modelo em um ambiente real de DP, ainda como um teste piloto, foi feita em uma subsidiária brasileira de uma empresa multinacional de máquinas e equipamentos para o setor agroindustrial. Nessa aplicação, foram visitados os setores envolvidos com o DP da referida empresa e realizada uma dinâmica de grupo com dois experientes engenheiros-projetistas, que já lideraram vários projetos de DP na empresa. Os resultados encontrados mostram a efetividade da ferramenta em avaliar detalhadamente a situação em que se encontra a GC no DP, e particularmente em quais dimensões do DP há maior intensidade no trabalho com o conhecimento. Esse estudo de caso é detalhadamente exposto em Silva \& Rozenfeld (2001b).

Por fim, a aplicação mais extensa (diversas visitas com entrevistas individuais), completa (amplo e heterogêneo quadro de entrevistados) e detalhada (teste da versão definitiva) do modelo, é apresentada no presente artigo e foi feita junto ao processo de DP de uma montadora multinacional de caminhões e ônibus.

No estudo de caso foram entrevistadas dez pessoas, em amostra representativa da diversidade de competências/habilidades das pessoas envolvidas ou relacionadas com o DP da empresa. Os entrevistados podem ser divididos em dois grupos básicos, conforme seu nível hierárquico e experiência: diretoria/gerência, constituído por quatro pessoas e supervisão/consultoria interna/coordenação, em que se enquadram seis pessoas.

É importante mencionar que as entrevistas não foram realizadas primeiramente com as pessoas pertencentes a um grupo e depois com as do outro grupo. As entrevistas foram feitas aleatoriamente, alternando pessoas dos dois grupos, conforme a disponibilidade de agenda dos entrevistados.

A Tabela 2 permite visualizar a abrangência da avaliação da GC realizada no processo de DP da empresa do estudo de caso, mostrando a ocorrência das hipóteses e melhores práticas nas quatro dimensões (do modelo esquematizado anteriormente na Tabela 1), pela avaliação do entrevistador/pesquisador das declarações dos entrevistados.

Tabela 2: Ocorrência das hipóteses e melhores práticas nas quatro dimensões.

\begin{tabular}{|c|c|c|c|c|c|c|c|c|}
\hline \multirow{3}{*}{\begin{tabular}{|c|} 
DIMENSÕES \\
Estratégia \\
(20/43) \\
$47 \%$
\end{tabular}} & \multicolumn{2}{|c|}{ SOCIALIZAÇÄO } & \multicolumn{2}{|c|}{ EXTERNALIZAÇÃOO } & \multicolumn{2}{|c|}{ COMBINAÇÃOO } & \multicolumn{2}{|c|}{ INTERNALIZAÇÃOO } \\
\hline & 9HТ 1MP & $1 \mathrm{HT}$ & ЗНТ 2MP & GHT & 3HT & 6HT 1MP & 2HT & 8HT 1MP \\
\hline & \multicolumn{2}{|c|}{$\begin{array}{c}(10 / 11] \\
91 \%\end{array}$} & \multicolumn{2}{|c|}{$\begin{array}{c}{[5 / 11]} \\
45 \%\end{array}$} & \multicolumn{2}{|c|}{$\begin{array}{l}3 / 10) \\
30 \%\end{array}$} & \multicolumn{2}{|c|}{$\begin{array}{c}{[2 / 11]} \\
18 \%\end{array}$} \\
\hline \multirow{2}{*}{$\begin{array}{c}\text { Organiz. } \\
\text { (42/61) } \\
69 \%\end{array}$} & 11НТ 6MP & $1 \mathrm{HT}$ & $5 \mathrm{HT} 4 \mathrm{MP}$ & 6НT 2MP & 4HT 1MP & 4HT 1MP & 8НТ ЗМР & 4HT 1MP \\
\hline & \multicolumn{2}{|c|}{$\begin{array}{c}(17 / 18) \\
94 \%\end{array}$} & \multicolumn{2}{|c|}{$\begin{array}{c}(9 / 17) \\
53 \%\end{array}$} & \multicolumn{2}{|c|}{$\begin{array}{c}(5 / 10) \\
50 \%\end{array}$} & \multicolumn{2}{|c|}{$\begin{array}{c}(11 / 16) \\
69 \%\end{array}$} \\
\hline \multirow{2}{*}{$\begin{array}{c}\text { Ativ. / Inf. } \\
\text { (71/107) } \\
66 \%\end{array}$} & २२НТ २МР & $4 \mathrm{HT}$ & 21HT 1MP & $13 \mathrm{HT}^{*} 1 \mathrm{MP}$ & 17HT & $4 \mathrm{HT}$ & 7HT 1MP & $14 \mathrm{HT}$ \\
\hline & \multicolumn{2}{|c|}{$\begin{array}{c}(24 / 28) \\
86 \%\end{array}$} & \multicolumn{2}{|c|}{$\begin{array}{c}(22 / 36] \\
61 \%\end{array}$} & \multicolumn{2}{|c|}{$\begin{array}{c}(17 / 21) \\
81 \%\end{array}$} & \multicolumn{2}{|c|}{$\begin{array}{c}\text { [8/22] } \\
36 \%\end{array}$} \\
\hline \multirow{2}{*}{$\begin{array}{c}\text { Recursos } \\
\text { [36/61] } \\
59 \%\end{array}$} & 8НТ ЗМР & $1 \mathrm{HT}$ & 10НТ 3МР & $9 \mathrm{HT}^{*}$ & ЗНТ २МР & GHT & $4 \mathrm{HT} 3 \mathrm{MP}$ & 9HТ \\
\hline & \multicolumn{2}{|c|}{$\begin{array}{c}(11 / 12) \\
92 \%\end{array}$} & \multicolumn{2}{|c|}{$\begin{array}{c}(13 / 22) \\
59 \%\end{array}$} & \multicolumn{2}{|c|}{$\begin{array}{c}{[5 / 11]} \\
45 \%\end{array}$} & \multicolumn{2}{|c|}{$\begin{array}{c}(7 / 16) \\
44 \%\end{array}$} \\
\hline $\begin{array}{c}\text { Processo } \\
\text { (169/272) } \\
62 \%\end{array}$ & \multicolumn{2}{|c|}{$\begin{array}{c}(62 / 69) \\
90 \%\end{array}$} & \multicolumn{2}{|c|}{$\begin{array}{c}(49 / 86) \\
57 \%\end{array}$} & \multicolumn{2}{|c|}{$\begin{array}{c}(30 / 52) \\
58 \%\end{array}$} & \multicolumn{2}{|c|}{$\begin{array}{c}(28 / 65) \\
43 \%\end{array}$} \\
\hline
\end{tabular}

Obs. 1: HT = hipóteses; MP = melhores práticas

Obs.2: Célula em azul claro = ocorrência efetiva das HT e/ou MP; Célula em azul escuro = ocorrência deficiente ou sem informações para avaliar Obs. 3: 0 asterisco (*) significa que uma das HT contadas é de ocorrência nula

Obs.4: Calculado em cada uma das células da tabela: Percentual = cocorrência efetiva das HT e MP na célula dividido por total de HT e MP na célula] multiplicado por cem 
A percepção do pesquisador ao conduzir e interagir com os entrevistados no estudo de caso permite afirmar que estes estavam efetivamente entendendo e julgando relevantes as dimensões do DP, para a constituição e o desempenho desse processo na empresa. Esta interpretação significa, ao menos para o DP da empresa do estudo de caso, que as dimensões do modelo de avaliação fazem sentido e sua aplicação é válida para a finalidade a que se propõe, de diagnóstico da GC no referido processo.

Os próximos parágrafos discutem os resultados encontrados na Tabela 2, sempre tomando como base as teorias de DP e GC que dão sustentação à construção das dimensões/conversões, hipóteses/melhores práticas, do modelo, e em alguns trechos citando certas particularidades identificadas no estudo de caso que reforçam esses resultados.

Para a dimensão Estratégia, o percentual de hipóteses/ melhores práticas válidas mostra um patamar elevado para a Socialização (91\%), relativamente bem acima das demais conversões - como, por exemplo, os $45 \%$ da Externalização, que é o segundo mais elevado.

No que se refere à Socialização, essa situação pode ser entendida como adequada, devido às próprias características das perspectivas da Estratégia que, naturalmente, precisam de grande interação humana nas negociações e discussões. Além deste fato, geralmente válido para todas as empresas, há, no processo de DP do estudo de caso, um ambiente e uma cultura propícios para otimizar a referida conversão.

No entanto, esta situação não se configura como razoável no que diz respeito às demais conversões e, em especial, à Internalização. Considerando as já mencionadas características das perspectivas da Estratégia, é natural que as demais conversões fiquem em um patamar abaixo ao da Socialização, porém não deveriam estar em uma posição tão reduzida como a que foi observada na presente avaliação.

A situação geral das quatro conversões do conhecimento mostra também que a Estratégia é a dimensão mais distante do nível médio em que se encontra a GC no processo de DP, apresentando quinze pontos percentuais a menos, quando comparado o número de hipóteses/melhores práticas válidas da Estratégia, com o do processo de DP.

Na dimensão Organização, a Socialização apresenta um elevado percentual de hipóteses/melhores práticas válidas. Esta situação é esperada para a referida dimensão, que naturalmente está ligada e é dependente dos relacionamentos humanos (inerentes à Socialização). Além disto, há um ambiente e uma cultura propícios a esta conversão do conhecimento na empresa do estudo de caso e, em particular, ao incentivo do trabalho em grupo, e à autonomia e iniciativa individual de atuação na orga- nização do processo de DP.

Por esse motivo, espera-se que as outras conversões do conhecimento não tenham o mesmo peso da Socialização, o que foi efetivamente observado no estudo de caso realizado. No entanto, a ocorrência dessas conversões (Externalização, Combinação e Internalização) é melhor na dimensão Organização do que na Estratégia.

A dimensão Atividades/Informações possui atividades que são intensamente tratadas entre pessoas - o que constitui um potencial para a Socialização - mas, muitas vezes, também necessitam que seus resultados sejam documentados, organizados e consultados, criando um potencial para as demais conversões do conhecimento.

A Socialização e a Combinação apresentam, ambas, um bom patamar de hipóteses/melhores práticas válidas, o que contribui para colocar a GC das Atividades/ Informações em um nível idêntico ao da Organização - respectivamente $66 \%$ e $69 \%$. Considerando-se todas as atividades, praticamente não foi observado qualquer problema grave nessas conversões do conhecimento. A Combinação é elevada nesta dimensão, devido à típica característica de agrupamento de documentos de várias das atividades, que é bem exercida no presente processo de DP.

A Externalização ficou em uma posição mediana, dispensando maiores preocupações, o que é reforçado pela ausência de falhas graves nesta conversão, segundo o que foi verificado nas análises realizadas em cada atividade. No entanto, a Internalização possui uma situação razoavelmente desfavorável se comparada às demais conversões, em especial às duas primeiras que mais se manifestam na referida dimensão. Nessa conversão se recomendam melhorias quanto aos incentivos para a leitura e estudo de registros e documentos diversos relacionados às atividades.

Em Recursos não se verificou a existência de falhas graves nas conversões do conhecimento e portanto em sua GC. A Socialização, em sintonia com o que foi observado nas outras dimensões, destaca-se significativamente tanto nos recursos que focalizam suas ações no apoio às discussões e decisões realizadas em grupo (notadamente o QFD, o DFMA e o FMEA), como em todos os recursos para transmitir "face a face" os aprendizados e experiências. A Externalização comporta uma maior ênfase na redação de aprendizados obtidos com o uso dos recursos, e há duas vertentes em que ações de melhoria, respectivamente na Combinação e na Internalização, devem se concentrar. Uma delas diz respeito à preocupação de se evitar a subutilização atual quanto ao potencial que estes recursos oferecem para 
apoiar e promover o agrupamento e o acesso aos documentos sobre o projeto de DP; a outra vertente refere-se ao incentivo (tempo e também cobrança) à leitura e ao estudo de registros e documentos diversos relacionados aos recursos.

As conversões do conhecimento foram inicialmente analisadas de forma particular para cada dimensão. Cabe agora fazer uma breve consideração quanto a estas conversões considerando o processo de DP. menor ocorrência no processo de DP. Se de um lado é aceitável seu patamar de ocorrência nas dimensões Organização e Recursos, de outro há um percentual baixo nas Atividades/Informações e na Estratégia, destoando em muito das demais conversões nessas duas dimensões. A falta de tempo para a leitura (estudo) e a preferência pela busca de conhecimentos via contatos com outras pessoas são as principais causas detectadas para esta deficiência, identificadas no DP da empresa do estudo de caso.

Ainda analisando alguns aspectos relacionados às conversões do conhecimento no estudo de caso, percebese que a transmissão de experiências e práticas do processo de DP se dá quase totalmente pela via tácita e, portanto, é fortemente dependente da Socialização, com vantagens (facilidade e rapidez) e desvantagens (monopólio individual do conhecimento e perda da memória corporativa). Atuar nas causas deste problema significa criar condições - especialmente preparo e tempo - para que as pessoas

A Socialização é a conversão do conhecimento com maior ocorrência no processo de DP, elevada e bem equilibrada entre as quatro dimensões. Estes resultados confirmam a percepção de que existe na empresa estudada um ambiente favorável à Socialização, resultado de ações empreendidas nos últimos anos para uma melhor comunicação e trabalho "face a face" entre as pessoas envolvidas com o DP (como, por exemplo, o incentivo e a preparação para o trabalho em grupo, etc.).

A Externalização possui uma posição intermediária de ocorrência no processo de DP. Analisando a ocorrência efetiva das hipóteses e melhores práticas, esta conversão é ligeiramente mais presente nas dimensões Atividades/ Informações e Recursos, provavelmente devido às necessidades de documentação e registro que as ações nestas duas dimensões exigem.

A Combinação também possui uma posição intermediária de ocorrência no processo de DP, observando-se a ocorrência efetiva das hipóteses e melhores práticas. Esta conversão ocorre com mais intensidade na dimensão Atividades/Informações, o que é esperado devido ao aspecto "aglutinador de documentos" de várias das atividades. Há, no entanto, uma certa fraqueza desta conversão na Estratégia, onde seria razoável se encontrar um percentual de ocorrência efetiva próximo ao observado na Externalização.

A Internalização é a conversão do conhecimento com realizem mais este tipo de leitura/estudo (Internalização) e, principalmente, estejam preparadas e motivadas para registrar (Externalização) com mais regularidade e correção as experiências e práticas observadas.

A próxima seção apresenta as principais implicações e reflexões resultantes do trabalho com o modelo de avaliação da GC no DP.

\section{CONCLUSÕES}

A aplicação do modelo de avaliação pode ser considerada como bem-sucedida, uma vez que demonstrou a lógica utilizada em sua construção, baseada no uso das hipóteses e melhores práticas que atestam a existência das conversões do conhecimento nas dimensões do processo de DP, já que os estudos de caso, particularmente o aqui detalhado, propiciaram um ambiente favorável para que todas estas hipóteses e melhores práticas pudessem ser testadas. A validação obtida do modelo de avaliação proposto reforça a adequação do referencial teórico utilizado em sua construção, uma vez que ele é a base na qual se estruturou o modelo aprovado.

As respectivas hipóteses e melhores práticas das perspectivas da Estratégia e da Organização, das Atividades e dos Recursos, fizeram sentido ou foram compatíveis com o que foi observado na prática real do DP, o que colaborou para cumprirem seu papel de orientar o pesquisador (entrevistador) a ficar atento a 
pontos específicos em que poderiam ocorrer as conversões do conhecimento, nas dimensões do processo de DP. Desta forma, estas hipóteses e melhores práticas permitiram que o pesquisador fizesse um questionamento mais crítico do objeto de estudo, refinando sua avaliação sobre as conversões por meio da busca de evidências, no estudo de caso, que comprovassem cada hipótese ou melhor prática investigada.

Como todo trabalho de pesquisa, ao necessariamente seguir determinadas opções metodológicas, certas limitações são inerentes à proposta aqui apresentada.

Como conseqüência de seu foco avaliador, o modelo não traz procedimentos e roteiros detalhados sobre como proceder ou atuar para a melhoria da GC no DP. Ele sinaliza as lacunas e, portanto, o que a empresa precisa melhorar em sua GC no DP, mas não tem a preocupação de abordar em detalhes quais são os passos a seguir para se fazer isso. Por possuir um foco avaliador ou de diagnóstico, tal modelo busca tornar visível e hierarquizar as falhas e lacunas nas dimensões do DP em que se podem implementar melhorias na GC. Como fazer, em detalhes, estas melhorias, deve ser buscado em outras literaturas específicas - muitas, inclusive, citadas e, em parte, discutidas ao longo deste trabalho.

A contingência de se seguir determinadas linhas teóricas para cada tema (as conversões do conhecimento para a $\mathrm{GC}$ e as dimensões do processo para o DP), consideradas aqui como sendo as mais pertinentes para se estudar a GC no DP, faz que se coloque necessariamente em segundo plano outras abordagens que também poderiam trazer algumas contribuições. Minimiza-se esta limitação, por meio de uma vasta análise realizada em diversificadas referências bibliográficas, buscando-se entender e considerar as influências praticadas ou recebidas pelas linhas teóricas adotadas em outras linhas que também tratam da GC e do DP.

No que se refere às dimensões do DP, estas possuem várias perspectivas (da Estratégia e da Organização), e várias Atividades e Recursos, e portanto, em si, já constituem uma grande área de estudo, que comporta uma análise mais aprofundada ou particular das conversões do conhecimento que nelas ocorrem. As restrições de foco existentes no presente trabalho impedem que isso seja feito, já que a prioridade escolhida é o mapeamento da GC no todo (no processo de DP). No entanto, trabalhos futuros terão na atual pesquisa um ponto de partida para aprofundamentos em dimensões específicas.

Por fim, conhecimento é um termo de certa subjetividade, que é entendido conforme o referencial que cada pessoa está utilizando. Da mesma forma, entender o significado e perceber a ocorrência de cada uma das conversões do conhecimento depende da avaliação pessoal de cada um, de sua experiência e vivência, e de sua preocupação com a neutralidade. Reduz-se esta limitação dando-se um mesmo referencial para as pessoas julgarem a ocorrência da conversão do conhecimento no processo de DP: uma definição consistente e clara de cada conversão, e o emprego de uma terminologia usual e consolidada pelas pessoas que trabalham com esse processo, através das dimensões do DP, das hipóteses e das melhores práticas.

Essas limitações do presente trabalho, bem como os resultados obtidos e anteriormente discutidos e comentados, servem de inspiração para algumas propostas de trabalhos futuros, derivados do atual, que explorem melhor certas lacunas e proposições aqui apontadas.

O presente modelo de avaliação da GC no DP, traduzido no questionário para aplicação por meio da entrevista, poderia também ser transposto para um questionário aplicado via survey, simplificando-o e adequando-o para o entendimento do respondente sem a presença do pesquisador, testando inclusive a possibilidade de avaliações quantitativas, por meio da escala Likert, para a mensuração da forma de ocorrência das conversões do conhecimento.

Outro caminho a ser explorado é uma análise mais profunda no DP de uma única empresa, porém indo além do que já foi feito atualmente por meio do estudo de caso. A pesquisa-ação (THIOLLENT, 1998), com a completa imersão do pesquisador no objeto de estudo - o processo de DP - em tempo integral e por longo prazo, e talvez acompanhando um projeto de um novo produto, poderia trazer relevantes contribuições na melhoria do modelo, notadamente pela investigação profunda de suas hipóteses e melhores práticas, assim como pela proposição de inúmeras outras.

$\mathrm{O}$ aspecto mais relevante das propostas anteriores de trabalhos futuros é que a originalidade do modelo de avaliação da GC no processo de DP cumpriu seu papel de abrir um novo nicho ou segmento de estudo, longe de se esgotar e com inúmeras possibilidades de novas contribuições.

\section{Edição especial \\ início $07 / 03 / 2003$ \\ fim $25 / 08 / 2003$}




\section{- Referências Bibliográficas}

APQP - ADVANCED PRODUCT QUALITY PLANNING. QS 9000: Quality System Requirements for Automotive Industry. USA, Omnex.

BABA, Y., NOBEOKA, K. Towards knowledge-based product development: the 3-D CAD model of knowledge creation. Research Policy, v. 26, n. 6, p. 643-659, 1998

BOLISANI, E., SCARSO, E. Information technology management: a knowledge-based perspective. Technovation, v.19, n.4, p.209-217, 1999.

BROWN, J. S.; DUGUID, P. Balancing act: how to capture knowledge without killing it. Harvard Business Review, v. 78 , n. 3, p. 73-80, MayJune, 2000.

CARRILLO, J. E.; GAIMON, C. Improving manufacturing performance through process change and knowledge creation. Management Science, v. 46, n. 2, p. 265-288, Feb., 2000.

CLARK, K., FUJIMOTO, T. Product Development Performance: strategy, organization and management in the world auto industry. Boston: Harvard Business School Press, 1991.

CLARK, K., WHEELWRIGHT, S. C Revolutionizing Product Development: quantum leaps in speed, efficiency, and quality. New York: The Free Press, 1992.

CLAUSING, D. Total Quality Development: a step-by-step guide to world-class concurrent engineering. New York: American Society of Mechanical Engineering Press, 1994
CLIFFE, S. Knowledge management - the well-connected business. Harvard Business Review, v. 76, n. 4 , p. 17-21, July-Aug., 1998.

CUSUMANO, M., NOBEOKA, K. Thinking Beyond Lean: how multiproject management is transforming product development at Toyota and other companies. New York: Simon \& Schuster, 1998.

DANE, F. C. Research Methods. Belmont, California: Brooks/Cole, 1990.

DAVENPORT, T. Process Innovation. Boston: Harvard Business School Press, 1994.

DRUCKER, P. The coming of the new organization. Harvard Business Review, v. 66, n. 1, p. 45-53. Jan-Feb., 1988.

EL SAWY, O. A. Understanding shared knowledge creation spaces around business processes: precursors to process innovation implementation. International Journal of Technology Management, v. 22, n. 1-3, p. 149-173, 2001.

HARMSEN, H. Company competencies as a network: the role of product development. The Journal of Product Innovation Management. v. 17, n. 3, p.194-207, May, 2000.

HAYES, R. H.; PISANO, G. P. Beyond world-class: the new manufacturing strategy. Harvard Business Review, v. 72, n. 1, p. 77-86, Jan-Feb., 1994

KLEINER, A., ROTH, G. How to make experience your company's best teacher. Harvard Business Review, v. 75, n. 5, p. 172-177, September-October, 1997.

LAKATOS, E. M., MARCONI, M. A. Fundamentos de Metodologia Científica. São Paulo: Editora Atlas, 1995.
LEONARD, D. Wellspring of Knowledge. Boston : Harvard Business School Press, 1995.

LIEBOWITZ, J. Knowledge Management Handbook. USA: CRC Press, 1999.

NONAKA, I., TAKEUCHI, H. Criação de Conhecimento na Empresa. Rio de Janeiro: Campus, 1997.

PRASAD, B. Concurrent Engineering Fundamentals: integrated product and process organization, v.1. New Jersey: Prentice Hall International Series, 1996.

PRASAD, B. Concurrent Engineering Fundamentals: integrated product and process organization, v. 2 . New Jersey: Prentice Hall International Series, 1997.

PUGH, S. Creating Innovative Products Using Total Design: the living legacy of Stuart Pugh. Massachusetts: Addison-Wesley, 1996

QUINN, J. B. Strategic outsourcing leveraging knowledge capabilities. Sloan Management Review, v. 40, n. 4 p. 9-21, Sum., 1999.

ROBERTSON, D., ULRICH, K. Planning for product platforms Sloan Management Review, Summer, v. 39, n. 4, p. 19-31, 1998.

ROZENFELD, H. (org.) Fábrica do Futuro: entenda hoje como sua indústria vai ser amanhã. São Paulo: Editora Banas, 2000.
SILVA, S. L. Proposição de um Modelo para Caracterização das Conversões do Conhecimento no Processo de Desenvolvimento de Produtos. Tese (Doutorado) - Escola de Engenharia de São Carlos, Universidade de São Paulo, 2002.

SILVA, S. L., ROZENFELD, H. Construção de um modelo para avaliação da gestão do conhecimento no desenvolvimento do produto. Anais em CD ROM do Congresso Brasileiro de Engenharia Mecânica (COBEM), Uberlândia, MG, 2001 a.

SILVA, S. L., ROZENFELD, H Proposta de uma ferramenta de diagnóstico da gestão do conhecimento no processo de desenvolvimento do produto. Anais em CD ROM do III Congresso Brasileiro de Gestão do Desenvolvimento de Produto (CBGDP), Florianópolis, SC, 2001b.

SOBEK II, D. K., LIKER, J. K., WARD A. C. Another look at how Toyota integrates product development. Harvard Business Review, v.76, n.4 p.36-49. July - Aug., 1998.

SPENDER, J. C. Making knowledge the basis of a dynamic theory of the firm. Strategic Management Journal, v. 17 , special issue in SI, p.45-62, Dec., 1996.

SUTTON, R. I.; PFEFFER, J. Knowing "what" to do is not enough: turning knowledge into action. California Management Review, v. 42, n. 1, p. 83 108. Fall, 1999.

THIOLLENT, M. Metodologia da Pesquisa-Ação. São Paulo: Cortez, 1998.

YIN, R. K. Case study research: design and methods. USA: Sage Publications, 1994. 